\title{
Mechanistic Materials Modeling for Nuclear Fuel Performance
}

\author{
Michael R Tonks ${ }^{\mathrm{a}, *}$, David Andersson ${ }^{\mathrm{c}}$, Simon R Phillpot ${ }^{\mathrm{d}}$, Yongfeng Zhang ${ }^{\mathrm{b}}$, Richard Williamson ${ }^{\mathrm{b}}$, \\ Christopher R Stanek ${ }^{\mathrm{c}}$, Blas P Uberuaga ${ }^{\mathrm{c}}$, Steven L Hayes ${ }^{\mathrm{b}}$ \\ ${ }^{a}$ Department of Mechanical and Nuclear Engineering, Pennsylvania State University, University Park, PA 16802. \\ ${ }^{b}$ Fuel Modeling and Simulation, Idaho National Laboratory, P.O. Box 1625, Idaho Falls, ID 83415. \\ ${ }^{c}$ Materials Science and Technology Division, Los Alamos National Laboratory P.O. Box 1663, Los Alamos, NM 87545 \\ ${ }^{d}$ Department of Materials Science and Engineering, University of Florida, Ganesville FL 32611
}

\begin{abstract}
Fuel performance codes are critical tools for the design, certification, and safety analysis of nuclear reactors. However, their ability to predict fuel behavior under abnormal conditions is severely limited by their considerable reliance on empirical materials models correlated to burn-up (a measure of the number of fission events that have occurred, but not a unique measure of the history of the material). Here, we propose a different paradigm for fuel performance codes to employ mechanistic materials models that are based on the current state of the evolving microstructure rather than burn-up. In this approach, a series of state variables are stored at material points and define the current state of the microstructure. The evolution of these state variables is defined by mechanistic models that are functions of fuel conditions and other state variables. The material properties of the fuel and cladding are determined from microstructure/property relationships that are functions of the state variables and the current fuel conditions. Multiscale modeling and simulation is being used in conjunction with experimental data to inform the development of these models. This mechanistic, microstructure-based approach has the potential to provide a more predictive fuel performance capability, but will require a team of researchers to complete the required development and to validate the approach.
\end{abstract}

Keywords: Fuel Performance Modeling, Multiscale Modeling and Simulation

\section{Introduction}

Currently $10.9 \%$ of the world's power is generated using light water nuclear reactors (LWRs) while $19.5 \%$ of power in the United States is generated using LWRs [1]. There are 439 operating nuclear reactors throughout the world, with 67 currently under construction [2]. Nuclear reactors have low greenhouse gas emissions, and are efficient, reliable and have low operating costs. However, while nuclear power has many benefits, their safety remains a major concern. While only three major accidents have occurred in commercial nuclear reactors over the fifty-five years of their operation, the results of such accidents can be catastrophic. Due to these safety concerns, detractors call for the shutdown of reactors across the world. However, the reality is

\footnotetext{
* Corresponding author

Email address: mrt5296@psu.edu (Michael R Tonks)
}

that there is not a current clean alternative that can take the place of nuclear power in the near future.

While nuclear power is guaranteed to continue to be used throughout the world for some time, changes are needed to improve the safety of nuclear reactors and to facilitate more flexibility in their operation. Current LWRs use stacked uranium dioxide $\left(\mathrm{UO}_{2}\right)$ fuel pellets encased by a zirconium alloy cladding. However, limitations of these materials can have catastrophic consequences during a reactor accident. Research is underway to determine new fuel concepts that can be used in existing LWRs that would allow more time after an accident thereby significantly reducing the risk of catastrophic consequences. These concepts include modifications to the zirconium alloy cladding to improve its corrosion resistance (including coatings), new cladding materials, and new fuel materials $[3,4]$. 
The power output of renewable resources are generally subject to the vagaries of the environment (wind turbines do not produce energy in still air; solar panels do not produce power at night), and these changes in production do not necessarily correspond with changes in demand. The current $\mathrm{UO}_{2}$ fuel and zirconium alloy cladding provide the best performance when power production remains as constant as possible. However, changes could be made to the current LWR fuel to increase the flexibility of nuclear reactors, allowing LWRs to increase power production when needed.

Improving the safety and flexibility of nuclear reactors will require improvement of the $\mathrm{UO}_{2}$ fuel and zirconium alloy cladding, or the development of new fuel and cladding materials. All past fuel development was conducted using a primarily experimental/empirical approach that required many years of expensive research. This high cost and time commitment has limited the magnitude of modifications being made to the fuel and cladding system during the 55 years of LWR operation, providing only incremental improvements to reactor operation. More revolutionary benefits to improve safety and flexibility could be facilitated if we reduce the time and expense required for fuel and cladding development. A critical component to reduce the time and expense is to combine modeling and simulation with experimental development effort.

Modeling has played a role in fuel development since the initial design of nuclear reactors [5]. However, as computers have become more powerful, computer simulations have become an increasingly important tool for understanding fuel development. Fuel performance codes predict the thermomechanical behavior of the fuel and cladding system during steady reactor operation, as well as in accident conditions. They accomplish this by solving partial differential equations (PDEs) defining mechanical behavior and heat conduction across the fuel pellet and cladding geometry with boundary conditions appropriate for reactor operation. The PDEs require material properties for $\mathrm{UO}_{2}$ and zirconium alloys; however, due to changes in the material microstructure that occur because of the harsh reactor environment, these properties change with time. Thus, models are needed that define these material properties as a function of the temperature and the changing microstructure.

The relationship between reactor conditions, evolution of the microstructure, and change in the material properties is very complicated. To sim- plify their development, models have traditionally been empirical or semi-empirical in nature, with parameters fit to experimental property measurements of samples that have undergone exposure in reactor. The models are typically correlated to the temperature and the burnup, a measure of how much energy is extracted from a primary nuclear fuel source. Burnup is quantified either by the fraction of fuel atoms that have undergone fission in \%FIMA (fissions per initial metal atom) or as the actual energy released per mass of initial fuel in gigawatt-days/metric ton of heavy metal (GWd/tHM), or similar units. These empirical models can be used to accurately interpolate the material properties within the range of experimental conditions to which they were fitted. However, they cannot extrapolate outside of those conditions. In addition, the models rely on burnup to quantify the change in the microstructure; however, there is not a unique relationship between microstructure evolution and burnup. The evolution is also impacted by the initial microstructure, the fission rate, the temperature gradient, and more. Because of these limitations, fuel performance codes that rely on empirical models cannot be used to explore new operating conditions or new fuel systems. Thus, their capabilities to assist in the modification of existing LWRs to be more safe and flexible are limited.

A more predictive approach to modeling the evolution of the material properties requires fuel performance codes that employ materials models that are functions of critical aspects of the microstructure rather than simply the burnup. While this microstructure approach has many benefits, it is extremely difficult to develop experimentally because a detailed understanding of the complex microstructural interactions is required. However, simulation capabilities at the atomistic and mesoscale have advanced to the point where they can now be used in conjunction with experiments to inform the development of the materials models required for microstructure-based fuel performance modeling.

In this work we discuss this microstructure based approach to fuel performance modeling. We begin by giving a brief overview of fuel performance modeling and mention some existing models used to describe the evolution of the material properties. We then provide a more detailed overview of our suggested approach, and how the development of the individual models can be informed by simu- 
lations at the atomistic and mesoscales. We end by mentioning uncertainty quantification and validation of this approach. Tables in the appendix summarize the microstructure variables and microstructure/property relationships that could be used in this approach, showing how they could be investigated both experimentally and with simulation and showing their readiness.

\section{History of Fuel Performance Modeling}

\subsection{Governing equations and solution methods}

The key phenomena occurring in nuclear fuel depend on the local temperature, so the first and ongoing task in modeling fuel behavior is accurate calculation of temperature. The fuel temperature, however, is often strongly influenced by the fuel and cladding mechanical behavior (e.g., evolution of the fuel-cladding gap in LWR fuel). Thus the thermal and mechanical behavior are interdependent and, in some cases (e.g., gap closure), can be strongly coupled. Additionally, species diffusion within the fuel and cladding can have a strong influence on fuel behavior and lifetime. For these reasons, the general relations governing fuel behavior consist of conservation equations for energy, momentum and chemical species. Fuel performance modeling pioneers, including Rashid [6], Olander [5] and Lassmann [7-9] described these equations in a variety of forms in early publications. The comprehensive set of partial differential equations were recently summarized in Williamson et al. [10].

Solution methods vary and have included both numerical and combined numerical-analytical approaches in both finite difference $[9,11,12]$ and finite element [6] formulations. A common geometrical simplification is to approximate a fuel rod using a set of axisymmetric one-dimensional radial representations, that loosely communicate via an axially varying coolant boundary condition $[9,11,12]$; this approach is described as being 1.5 dimensional. Although this approach does not resolve localized phenomena, such as fuel pellet hourglassing, it has been successfully used for decades and is still in use today [13]. The FALCON code, which has been available for nearly two decades, uses a finite element method (FEM) approach and is applicable to either 2D axisymmetric or planar geometries [6]. More recently, BISON, another FEM based fuel performance code, has been developed which employs parallel computation and can be applied to large, complex prob-

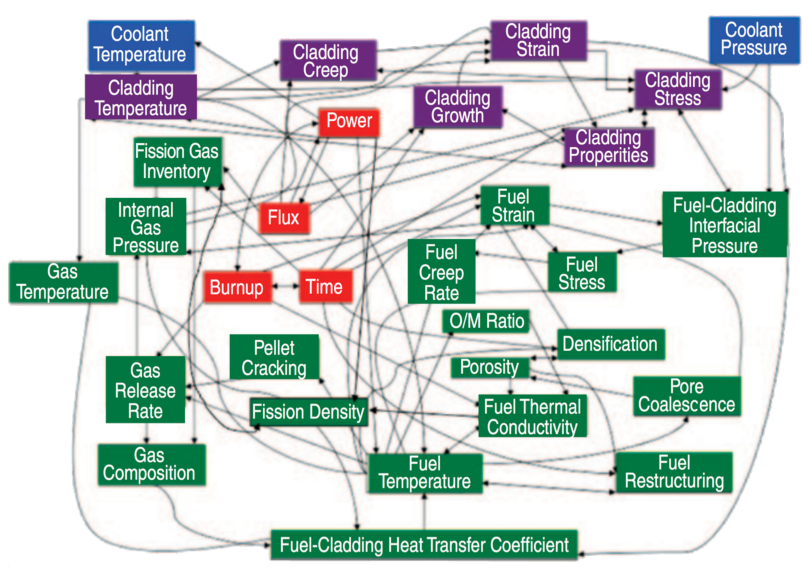

Figure 1: Complexity of reactor fuel rod behavior modeling. Taken from Rashid et al. [16] and adapted from Beyer et al. [17] and Lassmann [8].

lems in either 2D (axisymmetric or planar) or 3D $[10,14,15]$.

The material behavior within nuclear fuel is tightly coupled and very complex, as illustrated in Fig. 1. A large set of material and behavioral models are required to simulate this complicated behavior, as briefly summarized below. Note that we do not present a comprehensive review of the literature on materials models for fuel performance, but rather only give a short overview of key phenomena included in all fuel performance codes.

\subsection{Fuel material and behavioral models}

For the $\mathrm{LWR} \mathrm{UO}_{2}$ fuel, the key material models required are thermal conductivity and specific heat, swelling and densification, thermal and irradiation creep, fracture via relocation or smeared cracking, grain growth, and fission gas production, transport, and release.

\subsubsection{Thermal conductivity and specific heat}

Early models for fuel thermal conductivity involved empirical relations to describe dependence on temperature and porosity. However, as temperature comparisons were made to irradiated fuel, the importance of including effects from irradiation damage was recognized and correlations evolved to include dependence on burnup. Examples include the Fink-Lucuta approach which employs the Fink correlation for the temperature dependence of the thermal conductivity of the unirradiated material [18] and a series of corrections given by Lucuta to account for the effects of porosity and irradiation 
[19] and the NFIR correlation developed from thermal diffusivity measurements on specimens irradiated up to burnup levels of $80 \mathrm{MWd} / \mathrm{kgU}$ [20]. The specific heat is typically described using an empirical fit to temperature [18].

\subsubsection{Swelling and densification}

Fuel swelling due to solid fission products is typically expressed as a simple linear function of burnup. Historically, swelling from gaseous fission products was modeled using simple empirical functions of burnup and temperature. Recently, a physics-based model for the concurrent calculation of gaseous swelling and fission gas release has been developed as described in Pastore et al. [21]. The model incorporates a description of the underlying physical mechanisms of gas generation, diffusion, bubble growth and bubble inter-connection, and accounts for the inherent coupling between swelling and gas release. During initial irradiation some of the fabrication porosity disappears as a result of fission fragment impacts on pores. For simplicity this densification is typically accounted for using empirical correlations that specify how a fraction of the initial porosity is removed as a function of burnup and temperature.

\subsubsection{Fracture and relocation}

In $\mathrm{UO}_{2}$ fuel, a large temperature gradient develops from the fuel center to the radial edge. This gradient appears early and is strong enough to induce cracking in the fuel due to the associated stresses. Modeling fracture is important since cracks significantly reduce the stress state in the fuel and increase the effective fuel volume (i.e., decrease the fuel-cladding gap width). They also provide additional avenues for fission gas release. Two approaches are typically used to account for pellet fracture: relocation and smeared cracking. Relocation models are highly empirical and simply impose radial strains in the fuel to reduce the gap width, generally as a function of power and burnup. Smeared cracking models follow the approach originally outlined in Rashid [6], where cracking is simulated by adjusting the elastic constants at material points.

\subsubsection{Thermal and irradiation creep}

Thermal and irradiation creep processes are active in oxide fuel and are an additional source of stress relaxation during irradiation. A typical empirical model is provided in the MATPRO library and provides the combined thermal and irradiation creep strain rate as a function of time, temperature, effective stress, density, grain size, fission rate, and oxygen-to-metal ratio [22]. This model accounts for both diffusional and dislocation based thermal creep.

\subsubsection{Grain growth}

When a polycrystalline material experiences high temperatures and/or high irradiation dose, larger grains tend to grow at the expense of the smaller ones. Consequently, the smaller grains gradually disappear, reducing the total number of grains per unit volume and increasing the average grain size. The evolving granular structure of the fuel affects numerous physical processes. Grain growth in $\mathrm{UO}_{2}$ can be modeled using a simple empirical model [23] where the grain growth is specified via a rate constant and is subject to a limiting grain size.

\subsubsection{Fission gas production, transport, and re- lease}

The transport and release of fission gas generated in oxide fuel is typically computed by a two step process. Gas transport within fuel grains (assumed spherical) is computed via numerical solution of the diffusion equation in $1 \mathrm{D}$ spherical symmetry [24-26], with gas trapping into intra-granular bubbles and gas resolution from bubbles back into the lattice approximated by simple modifications to the diffusion coefficient. Consideration of gas behavior at grain boundaries includes calculation of both fission gas swelling and release through a direct description of the grain face bubble development. A modern physics-based approach which is simple enough for application in fuel behavior codes is described in detail in Pastore et al. [21]. Note that the prediction of fission gas release is subject to large modeling uncertainties as demonstrated in a recent uncertainty and sensitivity analysis [27].

\subsection{Cladding material and behavioral models}

For the zirconium alloy cladding, the key material models required are thermal and irradiation creep, irradiation growth, oxidation, and hydrogen transport/hydride formation.

\subsubsection{Thermal and irradiation creep}

During irradiation, Zircaloy cladding initially creeps inward due to the large pressure differential imposed by the reactor coolant. Once fuel-cladding 
gap closure occurs, cladding deformation reverses direction and the clad creeps outward in response to fuel swelling. Both thermal and irradiation creep mechanisms are active at typical operating conditions and are generally modeled using empirical relationships. The Limbäck model [28] is commonly used for primary and secondary thermal creep and provides the creep strain rate as a function of temperature, stress and time. Irradiation induced creep is often modeled using the Hoppe correlation [29] which relates the creep strain rate to stress and fast neutron flux.

\subsubsection{Irradiation growth}

Zircaloy undergoes irradiation-induced changes in dimensions in the absence of applied stress. In cladding this dimensional growth is principally in the axial direction. Empirical models are generally used to describe this behavior and simply prescribe the axial growth strain as a function of the fast neutron fluence. An example is the ESCORE model described in Rashid et al. [30].

\subsubsection{Oxidation}

Zircaloy cladding reacts with coolant water to form an oxide layer on the outer diameter. This oxide film can affect both the thermal and mechanical properties of the cladding. At normal operating temperature the oxide layer growth is calculated in two stages: a pre-transition process that follows a cubic time dependence up to a transition oxide thickness, and a post-transition process that follows a linear time dependence. As described in Gilmore et al. [31], rate constants can be defined in terms of coolant and cladding chemistry and fast neutron flux. At higher cladding temperatures (e.g., accident conditions) when the coolant begins to vaporize, oxidation proceeds at a much more rapid rate and can be described by a parabolic law with the reaction rate constant defined as a function of temperature through an Arrhenius relation [32].

\subsubsection{Hydrogen transport and hydride formation}

Concurrent with the oxidation process, hydrogen is absorbed into the cladding and diffuses under the influences of both temperature and stress. Due to the low solubility of hydrogen in Zircaloy, zirconium hydride precipitates form which are brittle and known to reduce the ductility of the cladding material. Models for hydrogen diffusion in Zircaloy under both mass and temperature gradients, including precipitation and dissolution of hydrides, are reported in Stafford [33].

\section{Multiscale Development of Microstruc- ture Based Materials Models}

The next generation of fuel performance codes needs to be able to accurately describe a range of different fuel and cladding materials and variable reactor operating conditions. This cannot be easily accomplished using empirical models correlated to burn-up, but requires a description of the material behavior based on changes in the microstructure. We propose that the optimal approach to creating a microstructure-based set of fuel performance models is to use internal state variables, as described in Tonks et al. [34]. In such an approach, the state of the microstructure throughout the fuel and cladding is described by locally varying variables, such as the average grain size and the dispersed fission gas concentration. These variables evolve with time as functions of the local temperature and stress, as well as other state variables, as defined by mechanistic materials models. The various material properties needed to define the fuel performance are determined using microstructure/property relationships that are functions of the operating conditions and the state variables, as illustrated in Fig. 2. Thus, the fuel performance of the fuel rod naturally evolves as the microstructure evolves.

As an example of this approach, we consider fission gas (see Fig. 3). The model presented in Pastore et al. [21] predicts the evolution of three distributions of fission gas: gas atoms distributed within the bulk of the fuel, gas trapped in intragranular bubbles, and gas in grain boundary bubbles. The gas atoms are produced due to the fissioning caused by the neutron flux, and their transport is influenced by the temperature, grain size, oxygen concentration, and uranium lattice point defects. In the model, the gas contained in grain boundary bubbles can eventually be released, raising the plenum pressure and lowering the gap thermal conductivity. However, these three distributions of fission gas also lower the thermal conductivity, the elastic modulus, and the fracture strength of the fuel. They also impact other mechanisms of microstructure evolution, such as fuel swelling, and grain growth. Thus, in our state variable approach, three separate state variables would be used to describe the fission gas: the concentration of dis- 


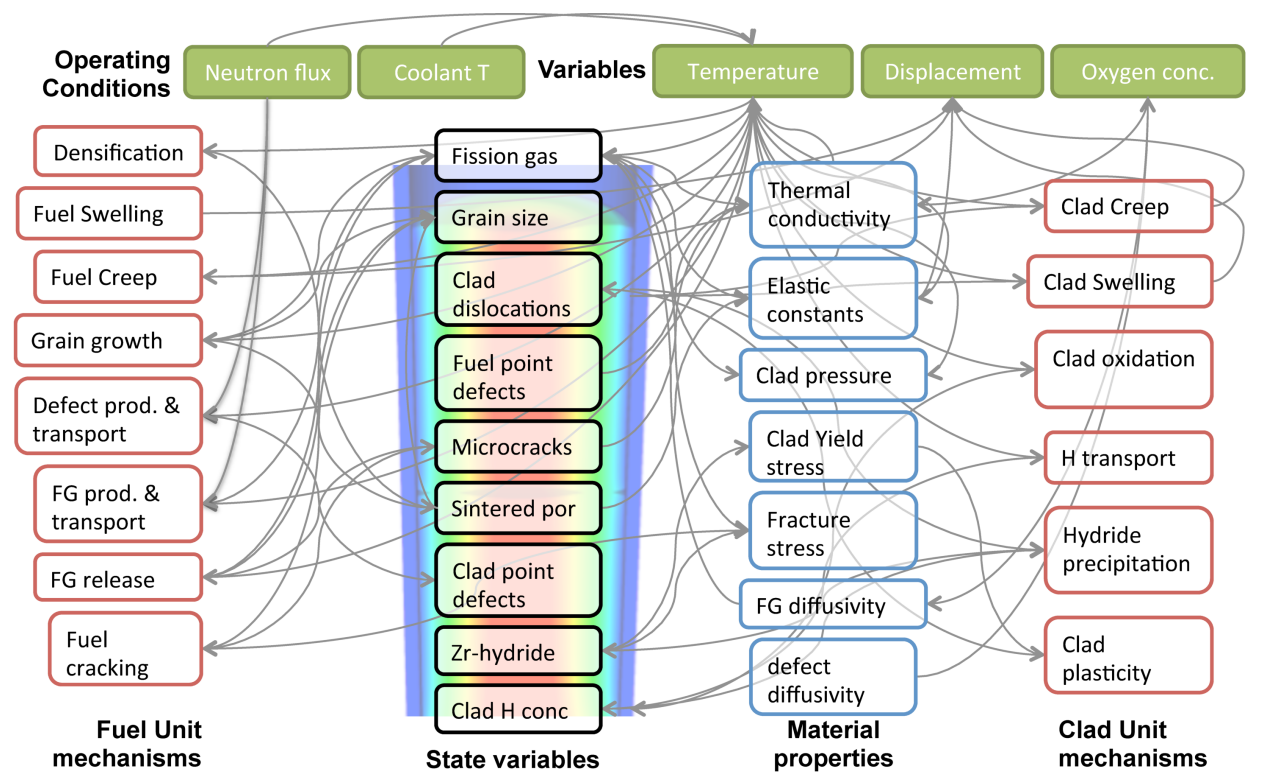

Figure 2: Illustration of the state variable approach suggested for fuel performance modeling to capture the complex behavior shown in Fig. 1. The local microstructure is characterized by the state variables, which evolve according to the unit mechanism models. The material properties are functions of the state variables. Note that the figure is not meant to be an inclusive list of all important phenomena.

tributed gas atoms, the volume concentration of intragranular gas bubbles, and the percentage of grain boundary area covered by gas bubbles. Their evolution is dictated by a unit mechanism model [21] that is a function of the neutron flux, the gas diffusivity (which in turn is a function of temperature, oxygen concentration, and uranium point defects) and grain size. Additional unit mechanism models are needed to define the impact of the fission gas variables on fission gas release [21], swelling [21], thermal conductivity [35], elastic constants, and fracture stress, as well as on the evolution of the grain size.

This microstructures-based approach using state variables provides a powerful tool for fuel performance modeling, but is much more difficult to develop than empirical models. The microstructure evolves in order to minimize the free energy of the system. Some of the evolution is a result of irradiation, but evolution also occurs due to elevated temperature, a large temperature gradient, and a corrosive environment. However, even in these cases, irradiation can modify the behavior. In order to develop unit mechanism models that define the evolution of each state variable defining the microstructure, it is necessary to distinguish the effects of irradiation from those of elevated temperature and

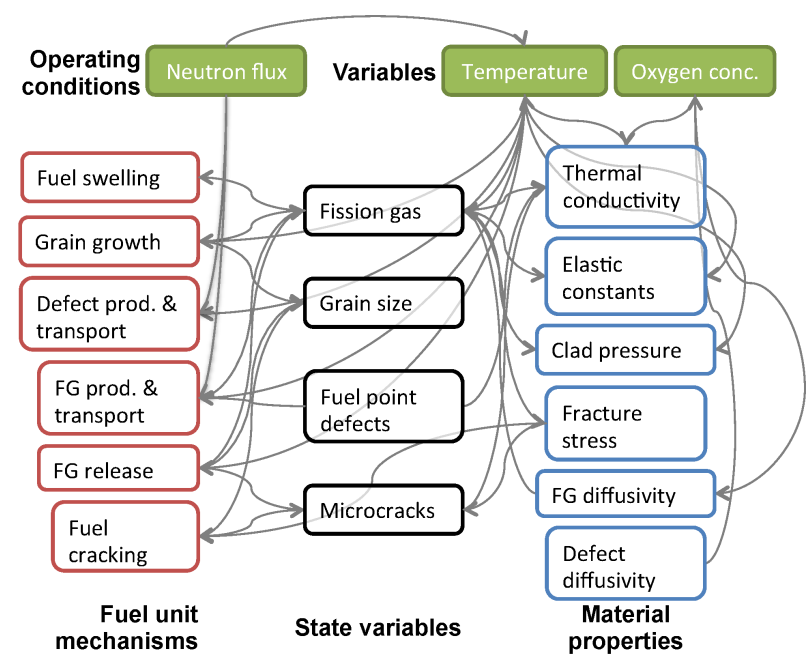

Figure 3: More detailed example of the state variable approach just regarding fission gas. 
elevated stress. Separating the effects in this way is very difficult to do experimentally, but is simpler using simulations at the atomistic and mesoscales. Thus, modeling and simulation at various length and time scales can be used along with experimental data to inform the development of evolution models for the state variables. Multiscale simulation also can play a critical role in determining the microstructure/property relationships for fuel performance.

In the following sections, we provide a brief overview of simulations that have been conducted at the atomistic and mesoscales to investigate microstructure evolution within the fuel and cladding. We also mention how these approaches are being used to inform the development of expressions that define the material properties as a function of the microstructure. In the appendix, we provide tables with potential state variables for the fuel and cladding and the experimental and simulation approaches that could be used to investigate them. We do the same for microstructure/property relationships in the fuel and cladding.

\section{Investigation of Fuel Behavior}

\subsection{Defect behavior}

The unique defect behavior of uranium dioxide plays an important role in its performance as a nuclear fuel. Improved predictability of performance and reliability models can be achieved though understanding the defects in $\mathrm{UO}_{2}$ and their transport. This includes both defects that are present before the fuel is introduced in the reactor and defects created during the fission process. At the most basic level, $\mathrm{UO}_{2}$ defect chemistry is typical of an ionic material, with anion Frenkel disorder being the predominant intrinsic defect process $[5,36]$. Uranium exhibits multiple charge states $\left(\mathrm{U}^{3+}, \mathrm{U}^{4+}, \mathrm{U}^{5+}\right.$ and $\mathrm{U}^{6+}$ ) and the fluorite phase is stable over a large compositional range [5], resulting in the formation of non-stoichiometric phases covering both the hypo- $\left(\mathrm{UO}_{2-x}\right)$ and hyper-stoichiometric $\left(\mathrm{UO}_{2+x}\right)$ ranges. Many properties of $\mathrm{UO}_{2}$ vary (sometimes strongly) as a function of $x$ in $\mathrm{UO}_{2 \pm x}$ (e.g. thermal conductivity, melting point, diffusion coefficients); thus it is critical that the evolution of the stoichiometry of the fuel be modeled in a microstructurebased fuel performance code. As an example, the strong variation of thermal conductivity and melting temperature as function of non-stoichiometry
$[37,38]$ affects the thermal margin and thus directly impacts the operational envelope of the fuel. In addition, the detailed defect structure of nonstoichiometric phases is itself complex and has received much attention [39-41]. Due to the rapid transport of oxygen defects and the critical impact of stoichiometry on many properties, stoichiometry is often treated as a solution variable along with the temperature and displacements [42-44].

Oxygen defects are responsible for the nonstoichiometry in $\mathrm{UO}_{2 \pm x}$ and typically occur in much higher concentrations than uranium defects. Compared to their uranium counterparts, oxygen defects also diffuse very rapidly [45]. However, microstructure evolution is often controlled by the slowest moving species in the lattice (as governed by their concentration and migration properties), which gives uranium defects, and in particular uranium vacancies, a prominent role. Defects on the uranium sublattice are also important because of their ability to accommodate or trap large fission gas atoms, such as Xe, and other fission products [46-55]. The release of fission gas atoms from the fuel pellet involves binding additional uranium vacancies to the trap site, thus enabling diffusion by a vacancy mechanism $[47,48,53]$. The concentration of uranium vacancies, and consequently also the fission gas diffusion coefficient, depends strongly on the fuel non-stoichiometry $[48,53,56]$. Thus, uranium interstitial and vacancy concentrations are important state variables.

\subsection{Grain size}

Grain boundaries (GB) play a critical role in fuel behavior, acting as sinks for point defects, segregation sites for fission gas, and preferred fracture paths. Fresh fuel pellets typically have an average grain size of around ten microns; however, the high temperatures near the fuel center during reactor operation result in GB migration and grain growth, in order to decrease the total free energy. The GBs migrate at a rate governed by the temperature, such that the temperature gradient in the fuel pellet results in a gradient in the average grain size, where the grain size stays around ten microns on the outer edge but is larger near the pellet center. Due to this gradient, fission gas behavior, swelling, creep, and fracture all occur differently at the center. Thus, the average grain size is a critical state variable in the microstructure-based fuel performance approach. 
GB migration can be approximated using the equation

$$
v_{G B}=M_{G B}\left(\sum P_{D}-\sum P_{r}\right),
$$

where $v_{G B}$ is the GB velocity, $M_{G B}=M_{0} e^{-Q / k_{b} T}$ is the GB mobility (with the the mobility prefactor $M_{0}$, the activation energy $Q$, the Boltzmann constant $k_{b}$, and the temperature $\left.T\right), P_{D}$ represents the various driving pressures, and $P_{r}$ represents the various resistive pressures. A large range of different driving forces can contribute, depending on the conditions and materials, though the driving force to reduce the GB energy (or curvature driving force $P_{D}=\gamma_{G B} / R$, with the grain boundary energy $\gamma_{G B}$ and radius of curvature $R$ ) is always present. The change in the average grain size $\bar{D}$ can be approximated using Eq. (1) as

$$
\frac{d \bar{D}}{d t}=2 v_{G B}=2 M_{G B}\left(\sum P_{D}-\sum P_{r}\right) .
$$

This equation is a function of measurable quantities, the GB energy and mobility, and the driving and resistive pressures can be coupled to other state variables such as the intragranular bubble density.

The GB energy has been determined for special $\mathrm{UO}_{2}$ GBs using molecular dynamics (MD) simulations [57]; however, an expression is still needed that fully defines the GB energy for any misorientation and inclination. Determining the intrinsic GB mobility for various GBs is also possible using MD, though, due to solute drag, the intrinsic mobility is typically several orders of magnitude larger than the actual mobility. Mesoscale simulation provide a powerful tool for understanding the driving and resistive pressures. Grain growth in $\mathrm{UO}_{2}$ has been modeled at the mesoscale using the phase field model with great success [58-62]. Phase field and MD simulations have shown that the temperature gradient driving force can be neglected in $\mathrm{UO}_{2}$ reactor fuel [60, 63]. Further work is needed to investigate the importance of other driving forces. The phase field method has also been used to develop a statistical model of the resistive pressure due to porosity aligned on the GB, such as fission gas bubbles [62]. However, work is needed to determine the impact of the pores being dragged by GBs.

\subsection{Fission gas behavior}

Large fission gas atoms such as Xe have very low solubility in the fuel matrix, which drives formation of intra-granular fission gas bubbles, segrega- tion of gas atoms to grain boundaries and development of inter-granular bubbles that eventually form percolation networks leading to release of the fission gas. Existing fission gas release models account for these processes by a coupled set of reactiondiffusion equations, which are typically solved for a spherical grain geometry with boundary conditions capturing the percolation threshold and subsequent gas release $[21,24,25,64-67]$. The underlying time scale for fission gas evolution is determined by the diffusion rate of fission gas atoms in bulk $\mathrm{UO}_{2}$ coupling to microstructure evolution, especially the nucleation, growth and resolution rates of intragranular bubbles. Altogether, this is a very complex problem and, despite the success of early models, there are still many unknowns preventing accurate prediction of fission gas retention/release under varying fuel operating conditions or accident scenarios. However, modern fission gas release models [21] already model the evolution of various distributions of fission gas and provide a strong foundation for a state variable approach.

Efforts to develop new mechanistic fission gas release models based on multiscale simulations have focused on understanding the behavior of fission gas atoms and point defects in bulk $\mathrm{UO}_{2}[49,51-$ $55,68-92]$ as well as how their properties are influenced by fuel chemistry $[49,53,78,82]$ and irradiation conditions [73], segregation to grain boundaries $[72,93,94]$, simulation of percolation networks at grain boundaries [95], nucleation and growth of inter- and intra-granular bubbles [85, 96-103] and bubble resolution [100, 104-106]. Attempts to use lower length scale simulations of fission gas properties in fuel performance simulations have so far relied on hierarchical approaches where certain parameters in the engineering scale models, such as the fission gas diffusion rate, are informed by lower length scale simulations $[73,107]$. In the future, frameworks will be developed to provide a closer coupling between the microstructure state of the fuel and fission gas release, as already pursued in a few existing studies [108-112].

The basic fission gas diffusion properties are determined by the location of gas atoms in the lattice and the interaction with thermal and irradiation induced defects. Many studies employing density functional theory (DFT) (see Fig. 4 for an example) and empirical potentials have shown the large fission gas atoms such as Xe prefer uranium vacancy trap sites $[49,53,82]$, in most cases with additional oxygen vacancies though the de- 


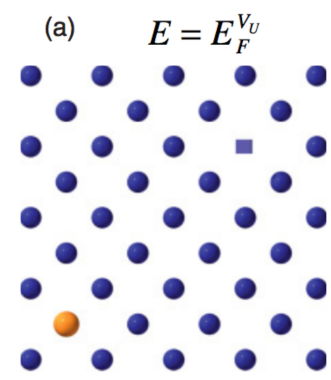

(c) $E=E_{F}^{V_{U}}-E_{B}$

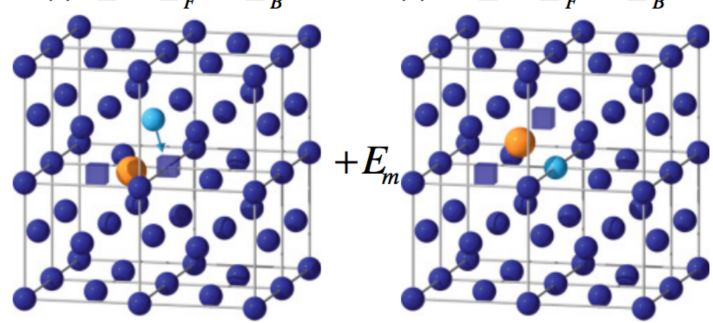

Figure 4: (Color online) Schematic illustration of Xe diffusion via a vacancy mediated mechanism used in DFT calculations. For simplicity the cubic $\mathrm{O}$ sublattice is omitted and only the fcc $U$ sublattice is shown. Xe atoms are shown in yellow, $\mathrm{U}$ in blue, and vacancies are represented by squares. (a) (100) projection of the U sublattice. (b) Xe occupying central position of two vacancies (c) Three-dimensional view of the fcc $\mathrm{U}$ sublattice with a Xe atom (d) Equivalent to the defect cluster in (c) but with the highlighted $U$ atom translated from its original position as an activated process associated with a migration barrier $E_{m}$, as indicated in the figure. Taken from Andersson et al. [53]

tailed balance is a function of non-stoichiometry $\left(\mathrm{UO}_{2 \pm x}\right)$ [53, 82]. Diffusion occurs through vacancy mechanisms involving at least one additional uranium vacancy bound to the trap site [53, 73]. The overall diffusivity is governed by the vacancy concentration, which is a function of the fuel nonstoichiometry, the irradiation conditions and the fuel microstructure determining the concentration of vacancy sinks, the binding energy between vacancies and the trap site and the cluster migration properties. Experimentally, the Xe bulk diffusion coefficient under irradiation is typically divided into three regimes, based on Turnbull's analysis [66]. At high temperature $(T>1650 \mathrm{~K})$ intrinsic diffusion dominates, while radiation-enhanced diffusion is the main contribution in the intermediate temperature range $(1381<T<1650 \mathrm{~K})$. Below 1381 $\mathrm{K}$, Xe diffusion is athermal and driven directly by irradiation damage processes. The intrinsic regime is fairly well understood, even though the diffusivity obtained from simulations is typically slightly underestimated compared to experiments [53, 73]. However, the irradiation-enhanced diffusivity is not accurately captured by the mechanisms described above, because the mobility predicted by DFT and empirical potential calculations for the defect clusters examined to date is too low. This discrepancy may be a consequence of neglecting the mobility of extended Xe-vacancy clusters, i.e. clusters involving more than one bound uranium vacancy and additional oxygen vacancies, or possibly Xe interstitials which are known to be highly mobile but also energetically very unfavorable [88].

\subsection{Fracture behavior}

$\mathrm{UO}_{2}$ is a ceramic, and is therefore brittle. When the reactor heats up, thermal stresses result in fracture via radial cracks that extend from the outer edge to roughly halfway through the radius. During reactor transients, further cracking occurs [5]. Fuel cracks have a significant effect on fuel performance because they result in a decrease in the gap size, restrict heat transfer, and provide avenues for fission gas release. Fission product generation and irradiation damage weakens the materials, decreasing the fracture strength. Thus, defining fuel cracks is essential to accurately describe behaviors such as fission gas release, but the cracking is also dependent on other microstructure variables such as the percentage of grain boundaries covered by fission gas bubbles (the fission gas fractional coverage).

Modeling and simulation has been used to investigate fracture in $\mathrm{UO}_{2}$ at the atomistic scale, the mesoscale, and the full pellet scale. Atomistic simulations using $\mathrm{MD}$ have been conducted to identify the fracture strength of a $\mathrm{UO}_{2}$ crystal [113]. Similar simulations have also been conducted to determine the fracture strength of various $\mathrm{UO}_{2}$ grain boundaries [114-116]. Such simulations tend to over-predict the fracture strength compared to experiments, because they use defect and impurity free materials. However, they do provide critical insights into the relative strength of the grain boundaries compared to the bulk.

Such atomistic characterization of the fracture strength can then be used in mesoscale simulations of polycrystalline material. A phase field model for fracture was recently parameterized for $\mathrm{UO}_{2}$ by comparing to MD simulation results [116]. This model represents a polycrystalline microstructure, with the fracture strength changing on grain boundaries versus the grain interior. Then, to determine 

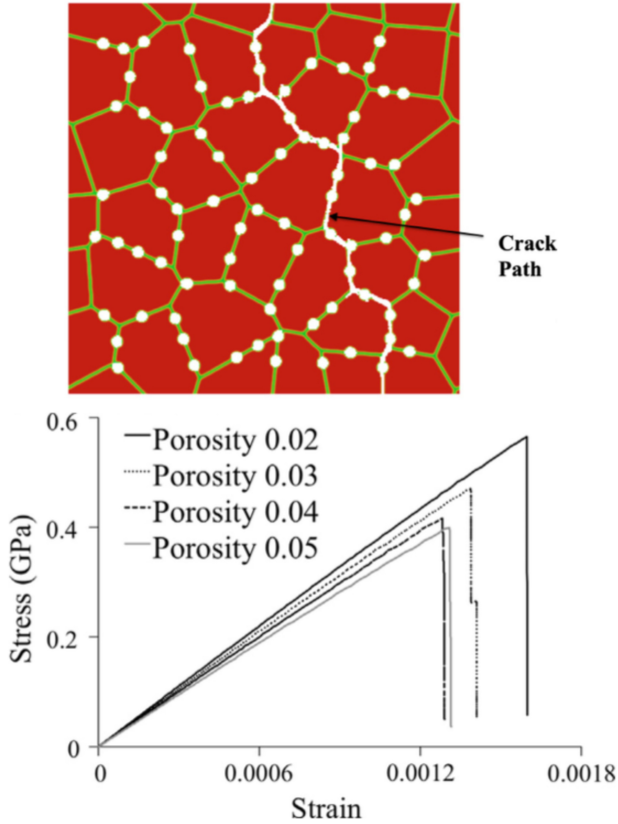

Figure 5: (Color online) Phase field simulation of fracture in porous $\mathrm{UO}_{2}$, where a microstructure with a porosity of $4 \%$ that cracked due to a uniaxial displacement controlled load is shown on the top. A comparison of stress-strain evolution along the primary loading direction for different porosities is shown below. These results provide the necessary information to create a unit mechanism model that connects fission gas state variables to the fracture stress. Taken from Chakraborty et al. [116].

the impact of fission gas bubbles along grain boundaries on fracture, increasing concentrations of bubbles were added to $2 \mathrm{D}$ polycrystal simulations, as shown in Fig. 5. Thus, a relationship between fission gas fractional coverage and fracture stress was obtained. This relationship can act as a unit mechanism model describing the impact of fission gas state variables on the fracture stress parameter. In addition, mesoscale finite element cohesive element simulations have been used to determine how bubble pressure can result in cracking and subsequent fission gas burst release [117].

While the lower lengthscale simulations provide necessary insights on the impact of microstructure on fracture, it is still critical to model discrete crack formation and propagation at the pellet scale. A pellet simulation employing cohesive elements has been used to model pellet fracture during reactor startup [118], as has the extended finite element method [119]. An alternative to finite element based simulation of cracking is to use a discrete body method such as the discrete element method. Such methods treat the fuel pellet as a rigid cluster of solid particles, and can naturally capture complex crack paths both during reactor startup and transients. Huang et al. [120] used such a method to illustrate that radial cracks occur as the temperature increases, and circumferential cracks occur as it decreases.

\subsection{Thermal conductivity}

An ideal fuel would have a very high thermal conductivity, which would result in a uniform temperature throughout and would thus enable a higher operating temperature and a higher resulting Carnot efficiency. However, single crystal $\mathrm{UO}_{2}$ has quite a low thermal conductivity, less than $10 \mathrm{~W} / \mathrm{mK}$. Point defects and large-scale microstructural elements lower this further. Moreover, fission radically evolves the chemical composition and leads to inhomogeneous thermal conductivity which decreases as the fuel burns up.

In order to replace empirical thermal conductivity models correlated to temperature and burnup, we must have a detailed understanding of the effects of all of the various microstructural features (e.g., fission products, grain boundaries, dislocations, voids, cracks) on the thermal conductivity. While such detailed information is difficult to obtain experimentally, atomistic and mesoscale simulations have been used with some success.

A prerequisite for a quantitatively accurate description of thermal transport is a correct description of the phonons which transport heat. Recently Pang and coworkers [121] have determined the phonon dispersion and lifetimes in $\mathrm{UO}_{2}$ and compared them with DFT predictions, showing that DFT accurately predicts the phonon behavior at low temperature $(300 \mathrm{~K})$, but not high $(1200$ $\mathrm{K})$. MD provides a direct means of calculating the thermal conductivity, though it drastically overpredicts the value [122-124]. Recent work indicates that this over-prediction is a result of resonant scattering of phonons by spins in the paramagnetic phase of $\mathrm{UO}_{2}[125,126]$ which is not captured by MD. This deficiency can be corrected by fitting a term to account for the spin scattering [35]. MD simulations have investigated the impact of the irradiation microstructure, including point defects [127, 128], grain boundaries [124], dislocations [129]; and voids [130]. In addition, there is a significant amount of variation in the thermal behavior predicted by various empirical potentials [123]. 

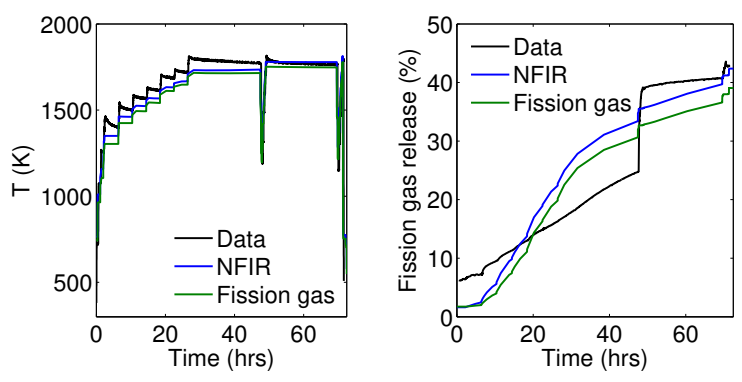

Figure 6: (Color online) Predicted centerline temperature (left) and fission gas release (right) for the Ris $\varnothing$ AN4 test case using the NFIR empirical thermal conductivity model [20] and a mechanistic thermal conductivity model account for the impact of fission gas. Note that the centerline temperatures predicted by the empirical NFIR model and the mechanistic fission gas model are nearly identical, though the mechanistic model was not fit to any integral fuel rod data. Taken from Tonks et al. [35].

Larger microstructural features can be investigated by solving the heat equation across a domain in which the thermal conductivity varies locally. These local changes in the thermal conductivity represent various microstructural features, such as fission gas bubbles, grain boundaries, and metallic precipitates. This approach has been used extensively to look at various types of fission products in 2D and 3D [131-136]. In a recent work [35], results from atomistic and mesoscale simulation, as well as analytical models, were combined to describe the impact of fission gas on the fuel thermal conductivity. In this work, a mechanistic thermal conductivity model focused on the impact of gaseous fission products was developed and coupled to a model of fission gas release (see Fig. 6 for an example of fuel performance predictions using this model). The fission gas release model evolved various state variables (as shown in Fig. 3), which impacted the swelling (using the model from Pastore et al. [21]) and the thermal conductivity using the new mechanistic model. The model was incomplete, as it only accounted for the impact of fission gas on the thermal conductivity and neglected point defects and other fission products, yet it performed reasonably well when compared against an empirical model.

\section{Investigation of Cladding Behavior}

\subsection{Oxidation}

The most common zirconium alloys in use for cladding materials of LWRs are ZIRLO ${ }^{\circledR}$ and $\mathrm{M} 5^{\mathrm{TM}}$, which have largely replaced Zircaloy4. These alloys have high concentrations of $\mathrm{Zr}$ $(>97 \%)$, with various alloying elements added to increase corrosion resistance [137]. Although the clad is subject to intense radiation in the reactor, the corrosion behavior is only weakly dependent on irradiation damage. Rather, the time and temperature in the water environment and the water chemistry are the dominant determinants of the macroscopic corrosion behavior [137]. The oxidation reaction itself is quite simple:

$$
\mathrm{Zr}+2 \mathrm{H}_{2} \mathrm{O} \rightarrow \mathrm{ZrO}_{2}+2 \mathrm{H}_{2}
$$

This reaction results in the formation of zirconia and hydrogen ingress into the zirconium alloy and the formation of zirconium hydride. The oxide layer impacts the thermal conductivity of the cladding, as well as its mechanical behavior, and therefore its thickness is a critical state variable.

The simulation of the oxidation process is very challenging because the kinetics are largely determined by the alloying elements and by the water chemistry. For example, the oxide layer in pure zirconium flakes off during operation while in the zirconium alloys it is protective. Thus simple studies of generic compositions, of which pure $\mathrm{Zr}$ is the simplest, can have only limited value in determining kinetics. However, because the fundamental oxidation chemical reaction is the same in the alloys, simple studies can provide value in characterizing oxidation mechanisms. For example, a simple DFT study of the oxidation of single crystal $\mathrm{Zr}$ with various surfaces exposed [138] showed an anisotropy in the oxygen adsorption, which is consistent with experiment [139]. In addition, first principles simulations have been applied to determine phase stability in the Zr-O system [140].

Attempts at modeling the cladding oxidation have also taken place at the mesoscale. The $\mathrm{C} 4$ oxidation model [141] is a one-dimensional mechanistic model of cladding oxidation that rationalizes the oxidation rate and hydrogen pickup in terms of oxide electronic conductivity. It predicts the oxidation kinetics of non-precipitate forming alloys with excellent accuracy using only the diffusion parameters and thermodynamic equilibrium concentrations of species at the interfaces as inputs. How- 
ever, it does not account for the impact of metallic precipitates on the electronic conductivity.

There remain enormous challenges to developing atomic scale models of key oxidation processes and incorporating them into mesoscale models. These include the transport of oxygen ions through pure $\mathrm{Zr}$ and through $\mathrm{Zr}$ with a finite concentration of dissolved oxygen interstitials, the diffusion of oxygen through the zirconium oxides [142], and the crystallography of zirconium dioxide.

\subsection{Hydrogen transport and hydride formation}

During fuel operation, a fraction of the total hydrogen produced by the reaction in Eq. 3 is picked up by the Zr-based cladding. This hydrogen transports through zirconium and forms hydride precipitates when the concentration is above the solubility limit [143-145]. Zirconium hydride is brittle, and the hydrides reduce the overall fracture toughness of the cladding [146]. Hydrides precipitate normal to the principle stress direction, and when oriented radially in the cladding serve as preferred fracture paths [147]. Even at stress levels far below the cladding fracture stress, hydrides can also cause cladding failure during used fuel storage due to delayed-hydride-cracking [148].

Models must predict the hydride size, concentration and orientation in order to predict their impact on the properties of the cladding. State variables defining the hydride microstructure could include hydride size, concentration and fraction of radial versus circumferential hydrides. Models would also be needed to relate these state variables to the mechanical properties of the cladding. To inform the development of these models, mesoscale simulations of hydride nucleation and formation are needed, with the required thermodynamic and kinetic information provided by atomistic calculations.

At the atomic scale, DFT calculations and MD simulations have been applied to obtain the thermodynamic stabilities of hydrogen in solid solution and hydride phases, the hydrogen diffusivity, and the hydride formation mechanisms [149-153]. Hydrogen atoms are predicted to diffuse by interstitialinterstitial jumps [149], with barriers close to the effective barrier measured by experiments [154]. The formation energies of various hydride phases predicted by DFT calculations [149-153, 155, 156] were also in good agreements with experimental results [157]. In addition to the four experimentally observed hydride phases, $\epsilon\left(\mathrm{ZrH}_{2}\right), \delta\left(\mathrm{ZrH}_{\sim 1.6}\right)$, $\gamma(\mathrm{ZrH})[158]$ and $\zeta\left(\mathrm{Zr}_{2} \mathrm{H}\right)[159]$, several metastable hydride phases were also predicted by DFT calculations or DFT-based thermodynamics analysis $[152,153,160,161]$. These metastabe phases are believed to be important for the nucleation process of hydrides. In line with a previously suggested nucleation mechanism [162], recent MD simulations demonstrated that the $\gamma$ hydride could form by shearing the $\zeta$ hydride [163]. A negligible barrier was identified for the shear, in agreement with previous DFT calculations [150]. These atomic scale studies provided important insights for mesoscale model development. As most of these works focused on single crystal $\mathrm{Zr}$ without irradiation and mechanical loading, the effects of stress, alloying elements, grain boundaries and irradiation damage on hydride evolution are yet to be explored. The mechanical properties of hydride phases are not fully clear either, though the effect of temperature and anisotropic stress on the elastic properties of the $\epsilon$ phase has been investigated using anharmonic potential cluster expansion within Monte Carlo simulations [164]. The mechanical properties of the hydride phase are needed to quantify the degradation in mechanical properties of claddings.

At the mesoscale, various phase field models (summarized in Motta and Chen [144], Bair et al. [145]) have been developed to describe the formation and evolution of different hydride phases including $\zeta$ [159], $\gamma[165]$ and $\delta$ [166]. However, these models need to be expanded using the mechanisms and parameters obtained from the atomic scale to predict hydride microstructure, including the volume fraction, crystal phases and sizes, and orientations as functions of the fuel operating history.

\subsection{Creep}

Due to the HCP crystal structure, the mechanical properties of Zr alloys are highly anisotropic. Deformation involves a combination of dislocation slip and twinning, where the dominant slip system is prismatic. In addition, the clad has a very carefully controlled microstructure [167]. In particular, to improve mechanical performance, increase corrosion resistance, and mitigate the detrimental effects of hydride formation, the material is generally highly textured with the c-axis lying at approximately $30^{\circ}$ to the surface.

There are surprisingly few studies of deformation processes associated with creep in hcp metals. Recently $\mathrm{Lu}$ et al. used atomistic simulation to examine Zr under tension [168] and nanoindentation [169] to characterize the deformation processes. 
They compared the predictions of MD simulations using an Embedded Atom Method (EAM) potential with those using a Charge Optimized Many Body (COMB) potential. In first examining the stacking fault $\gamma$-surfaces on the prismatic $[10 \overline{1} 0]$ plane, they found that the COMB potential reproduced the results from DFT better than the EAM potential [168]. Although this difference leads to differences in the predicted nature of prismatic slip, the overall mechanical response predicted by the two potentials is quite similar. This strongly suggests that the atomistic simulations are capturing the fundamental deformation behavior and thus can, at least in principle, provide input to mesoscale simulations.

Since the mechanical properties of the various $\mathrm{Zr}$ clad alloys differ, it is important to understand the effects of the minor components on the mechanical properties. However, at this time, there are no simulations of such alloyed systems.

Under irradiation, Zr exhibits significant changes in microstructure and macroscopic properties, the most prominent being so-called irradiation growth $[170,171]$. These irradiation-induced changes involve the nucleation and growth of both interstitial and vacancy loops. Predicting their effect on mesoscopic response of the clad involves deriving models of defect evolution [172] and coupling those to plasticity models [173]. One such approach was developed by Subramanian and Tome [174], based on a defect model developed by Golubov et al. [175]. These types of simulations couple the evolution of radiation damage with the creep behavior in clad. Ongoing work is focused on understanding the detailed atomistic behavior that dictates the nucleation and growth of these loops, accounting for the effects of strain on the migration of radiationinduced defects [176], as shown in Fig. 7.

\section{Validation and Uncertainty Quantifica- tion}

In fuel performance modeling, uncertainty arises from various sources including uncertainty in the operating conditions, in both the coolant conditions and the neutron flux, inaccurate physics models and incorrect coupling of physics, uncertainty in the material parameters, and numerical error in the solution of the governing equations. These uncertainties can be quite large in fuel performance simulations because in-pile data is difficult to obtain and our understanding of all the complicated

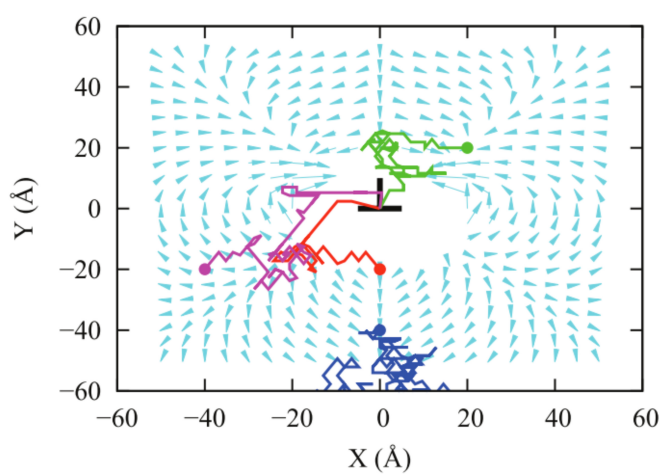

Figure 7: (Color online) The rate-weighted average displacement vector of a vacancy in $\mathrm{Zr}$, as a function of position, in the vicinity of an edge dislocation computed using kinetic Monte Carlo. Only a two-dimensional slice at $\mathrm{z}=0$ is shown. The vector shows the most likely direction of motion of the vacancy. Also shown are $2 \mathrm{D}$ projections of a few representative trajectories of the vacancy with their starting points marked by solid circles. Taken from Subramanian et al. [176]

phenomena that take place within the fuel during operation is still limited. For example, a deviation of a factor of two between calculated and measured fission gas release is commonly accepted [27]. Compared with the burnup based approach with empirical models, microstructure based fuel performance modeling as suggested in this work allows for the inclusion of richer physics and also more rigorous validation by comparing to microstructure data. As the physics from various scales and areas are integrated together to predict the materials properties, a multiscale, multiphysics validation practice is required to fully assure the validity of these models. This validation will facilitate a significant reduction in the inaccuracies in the individual models, and thus reduce the overall uncertainty. In addition, lower length scale simulations are helping to reduce the uncertainty of the material parameters needed for the individual models.

\subsection{Uncertainty quantification}

One necessary precursor to validation of the various models is the capability to propagate uncertainty from the materials parameters through the models to the final predicted behavior. In other words, it is not enough for the models to only predict the average behavior, they need to predict the overall distribution of that behavior as well. Uncertainty quantification can be accomplished using 
external tools that run multiple iterations of simulations while varying the parameters to estimate the propagation of uncertainty, or via internal calculations of variable gradients. The external tools are typically less numerically efficient but much more convenient as they do not require modification of the base code. For fuel performance modeling, external uncertainty quantification tools have been incorporated using the DAKOTA [27] and RAVEN codes [177]. During validation, uncertainties in the experimental data must also be considered to determine a final probability margin that the model is accurately representing the true physical behavior.

\subsection{Multiscale validations}

The validation of microstructure based fuel performance modeling must take two forms: validation of the lower length-scale models used to inform the macroscale materials models as well as validation of the fuel performance code against integral reactor data. The first effort focuses on the validation of the models representing individual physics and the second on the interaction between the unit mechanisms models that have been developed to describe the microstructure evolution and the structure-property relationships. Validation of the lower length-scale models is a difficult undertaking, due to the complexities in obtaining data at the requisite scales on irradiated materials. Thus, separate effects tests are employed to validate atomistic and mesoscale predictions on systems with irradiated or unirradiated materials. Each experiment investigates a single phenomena, such as oxidation of zirconium alloys or grain boundary migration in $\mathrm{UO}_{2}$. Validation of the lower length-scale models is critical for the multiscale approach to result in a predictive capability. In addition to the lower length-scale validation, the macroscale fuel performance code still must be validated in the traditional manner, by comparing to reactor data from various steady and transient irradiation conditions [178].

\section{Conclusions and Looking Forward}

Fuel performance simulations provide a critical tool for improving the safety and flexibility of light water reactors. However, predictive materials models are needed that are based on microstructure rather than burnup to describe the complex material interactions that take place within the fuel and cladding. We propose the development of a state variable approach, in which a series of microstructure variables describe the current state of the microstructure. The evolution of these variables is defined by the mechanistic materials models that are functions of the operating conditions and other variables, as shown in Fig. 2. The material properties, such as the elastic constants and thermal conductivity, are functions of the state variables, and thus evolve as the microstructure evolves. While such an approach has many benefits, it would be very difficult to develop using only experimental data. Therefore, we propose the application of simulation at the atomistic and mesocales to be used with data to develop these microstructurebased materials models. Potential state variables and microstructure/property relationships are summarized in tables in the appendix.

We have reviewed examples of lower length-scale simulation being applied to understand the microstructure evolution that takes place within reactor fuel and cladding and how it impacts the properties. However, this work is far from done if we are to develop a complete set of models describing this complex behavior. Detailed experimental data is also needed to validate these models. However, while the work discussed here has been applied to zirconium alloy cladding and $\mathrm{UO}_{2}$ fuel, other materials and concepts can be explored using this approach. Thus, libraries of materials models can be developed for a range of different materials to allow for the design of fuel and cladding combinations that provide revolutionary improvements in reactor safety and flexibility.

\section{Acknowledgements}

The majority of this work was supported by the US DOE Nuclear Energy Advanced Modeling and Simulation program. The work of SRP was supported by DOE NEUP 12-4728. This manuscript has been authored by Battelle Energy Alliance, LLC under Contract No. DE-AC07-05ID14517 with the US Department of Energy. The United States Government retains and the publisher, by accepting the article for publication, acknowledges that the United States Government retains a nonexclusive, paid-up, irrevocable, world-wide license to publish or reproduce the published form of this manuscript, or allow others to do so, for United States Government purposes. Los Alamos National Laboratory, an affirmative action/equal opportunity employer, is operated by Los Alamos National Security, LLC, 
for the National Nuclear Security Administration of the U.S. Department of Energy under Contract No. DE-AC52-06NA25396.

\section{Appendix: Summary of Proposed State Variables}

Here we provide summary tables regarding the state variables we propose for both the fuel (Table 1 ) and the cladding (Table 3 ). We also provide tables for the microstructure/property relationships that are required to connect the microstructure state variables to the various properties for the fuel (Table 2) and the cladding (Table 4). This is not a finalized list, and the need for additional state variables and microstructure/property relationships could arise. For each state variable, we show the readiness of the models for that variable, tools that could be used to model it, and how the variable could be characterized experimentally. Note that the fuel oxygen stoichiometry will be solved as a nonlinear variable over the entire fuel pellet, and therefore is not listed as a state variable.

To conserve space in the tables, a number of acronyms are used. The acronyms for simulation methods are: MD for molecular dynamics, DFT for density function theory, PFM for the phase field method, HC for heat conduction, SM for solid mechanics, KMC for kinetic Monte Carlo, RT for rate theory and cluster dynamics, and DD for dislocation dynamics. The acronyms for experimental methods are: OM for optical microscopy, SEM for scanning electron microscopy, EBSD for electron backscatter diffraction, TEM for transmission electron microscopy, PAS for positron annihilation spectroscopy, APT for atom probe tomography, EDS for energy dispersive X-ray spectroscopy, WDS for wavelength dispersive X-ray spectroscopy, EELS for electron energy loss spectroscopy, FIM for field ion microscopy, SIMS for secondary ion mass spectroscopy, ICPMS for inductively coupled plasma mass spectroscopy, TIMS for thermal ionization mass spectroscopy, EPMA for electron probe micro-analysis, RUS for resonant ultrasound spectroscopy, DTA for differential thermal analysis, DSC for Differential scanning calorimetry, DIL for dilatometry, XRD for X-ray diffraction, UT for ultrasonic testing, TT for tensile testing, CIT for Charpy impact test, CTT for compact tension testing, ECP for Eddy current probe, NI for nanoindentation, VM for volume measurement, and LF for laser flash. In addition, we abbreviate new methods needed with NMN. Finally, we abbreviate concentration with conc. and fraction with frac.

\section{References}

[1] Nuclear Energy Institute, Nuclear energy around the world, URL: http://www.nei.org/knowledge-center/, 2015.

[2] International Atom Energy Agency, Power Reactor Information System: URL: https://www.iaea.org/pris/, 2015.

[3] S. Bragg-Sitton, Nuclear News (2014) 83-91.

[4] S. J. Zinkle, K. A. Terrani, J. C. Gehin, L. J. Ott, L. L. Snead, Journal of Nuclear Materials 448 (2014) 374-379.

[5] D. R. Olander, Fundamental aspects of nuclear reactor fuel elements, Technical Information Center, Energy Research and Development Administration, 1976.

[6] Y. R. Rashid, Nuclear Engineering and Design 29 (1974) 22-32.

[7] K. Lassmann, Nuclear Engineering and Design 45 (1978) 325-342.

[8] K. Lassmann, Nuclear Engineering and Design 57 (1980) 17-39.

[9] K. Lassmann, H. Blank, Nuclear Engineering and Design 106 (1988) 291-313.

[10] R. L. Williamson, J. D. Hales, S. R. Novascone, M. R. Tonks, D. R. Gaston, C. J. Permann, D. Andrs, R. C. Martineau, Journal of Nuclear Materials 423 (2012) $149-163$

[11] G. A. Berna, C. E. Beyer, K. L. Davis, D. D. Lanning, FRAPCON-3: A Computer Code for the Calculation of Steady-State, Thermal-Mechanical Behavior of Oxide Fuel Rods for High Burnup, Technical Report NUREG/CR-6534 Vol. 2, PNNL-11513, Pacific Northwest National Laboratory, 1997.

[12] W. J. Kilgour, J. A. Turnbull, R. J. White, A. J. Bull, P. A. Jackson, I. D. Palmer, in: Proceedings of the ENS Meeting on LWR Fuel Performance, Avignon, France.

[13] H. S. Aybar, P. Ortego, Progress in Nuclear Energy 46 (2005) 127-141.

[14] B. W. Spencer, J. D. Hales, S. R. Novascone, R. L. Williamson, in: Transactions of Top Fuel Reactor Fuel Performance 2012, Manchester, United Kingdom.

[15] J. D. Hales, R. L. Williamson, S. R. Novascone, D. M. Perez, B. W. Spencer, G. Pastore, Journal of Nuclear Materials 443 (2013) 531-543.

[16] J. Y. Rashid, S. K. Yagnik, R. O. Montgomery, JOM 63 (2011) 81-88.

[17] C. Beyer, C. Hann, D. Lanning, F. Panisko, L. Parchen, GAPCON-THERMAL-2: a computer program for calculating the thermal behavior of an oxide fuel rod, ERDA, 1975.

[18] J. K. Fink, Journal of Nuclear Materials 279 (2000) $1-18$.

[19] P. Lucuta, H. Matzke, I. Hastings, Journal of Nuclear Materials 232 (1996) 166-180.

[20] A. Marion (NEI) letter dated June 13, 2006 to H. N. Berkow (USNRC/NRR), Safety Evaluation by the Office of Nuclear Reactor Regulation of EPRI TR-1002865, Topical Report on Reactivity Initiated Accidents: Bases for RIA Fuel rod Failures and Core Coolability Criteria, 
Table 1: Microstructure state variables for the fuel

\begin{tabular}{|l|l|l|l|}
\hline State Variable & Readiness & Modeling approach & Experimental approach \\
\hline 1. Average grain size & High & MD, PFM & OM, SEM, EBSD \\
2. Dislocation density & Low & MD, DD, PFM, RT & TEM, XRD, APT, FIM \\
3. U vacancy conc. & Medium & DFT, MD, RT, KMC & XRD, PAS \\
4. U interstitial conc. & Medium & DFT, MD, RT, KMC & XRD \\
5. Dissolved gas atom conc. & High & DFT, MD, RT, KMC & SIMS, ICPMS, APT, \\
& & MD, PFM, RT & EELS \\
6. Intragranular bubble conc. & Medium & SEM, TEM, EELS \\
7. Gas GB fractional coverage & Medium & PFM & OM, SEM \\
8. Pu conc. & Low & DFT, MD, PFM & EPMA, EDS, WDS, TIMS, \\
9. Metallic FP precipitate frac. & Low & DFT, PFM & APT, EELS \\
OMM, SEM, TEM, EPMA, \\
10. Ceramic FP precipitate frac. & Low & DFT, PFM & EELS \\
11. Dissolved FP conc. & OM, SEM, TEM, EPMA, \\
12. Sintered porosity & Low & DFT, MD, RT & EELS \\
13. Fracture parameter & Low & PFM & OM, SEM , EELS \\
\hline
\end{tabular}

Table 2: Property/microstructure relationships for the fuel. Note that the state variable numbers refer to Table 1

\begin{tabular}{|l|c|c|c|c|}
\hline Property & State vars. & Readiness & Modeling approach & Experimental approach \\
\hline Elastic constants & $1-13$ & Low & DFT, MD, SM & UT, RUS, NI \\
Thermal conductivity & $1-13$ & High & DFT, MD, HC & LF, DSC, VM, DIL \\
Fracture toughness & $1,2,7,12$ & Medium & DFT, MD, PFM, SM & CIT, CTT, NI \\
Melting temperature & $8,10,11$ & Low & DFT, MD, PFM & DTA, DSC, DIL \\
O Diffusion coefficient & $1-5,8,11$ & High & DFT, MD, KMC & NMN \\
Thermal exp. coef. & $6-10,12$ & High & DFT, MD, SM & DIL, XRD \\
Dimensional change & $6,7,9-13$ & Medium & PFM, SM & VM, DIL, XRD \\
\hline
\end{tabular}

http://pbadupws.nrc.gov/docs/ML0616/ML061650107.pdf, 2006 .

[21] G. Pastore, L. Luzzi, V. Di Marcello, P. Van Uffelen, Nuclear Engineering and Design 256 (2013) 75-86.

[22] C. M. Allison, G. A. Berna, R. Chambers, E. W. Coryell, K. L. Davis, D. L. Hagrman, D. T. Hagrman, N. L. Hampton, J. K. Hohorst, R. E. Mason, M. L. McComas, K. A. McNeil, R. L. Miller, C. S. Olsen, G. A. Reymann, L. J. Siefken, SCDAP/RELAP5/MOD3.1 Code Manual, Volume IV: MATPRO-A Library of Materials Properties for Light-Water-Reactor Accident Analysis, Technical Report NUREG/CR-6150, EGG-2720, Idaho National Engineering Laboratory, 1993.

[23] J. Ainscough, B. Oldfield, J. Ware, J. Nucl. Mater. 49 (1973) 117-128.

[24] A. Booth, A METHOD OF CALCULATING FISSION GAS DIFFUSION FROM UO sub2 FUEL AND ITS APPLICATION TO THE X-2-f LOOP TEST, Technical Report, Atomic Energy of Canada Ltd. Chalk River Project, Chalk River, Ont., 1957.

[25] M. Speight, Nuclear Science and Engineering 37 (1969) 180-185.

[26] M. Speight, W. Beere, Metal Sci. 9 (1975) 190-191.

[27] G. Pastore, L. P. Swiler, J. D. Hales, S. Novascone,
D. M. Perez, B. W. Spencer, L. Luzzi, P. V. Uffelen, R. L. Williamson, Journal of Nuclear Materials 456 (2015) 398-408.

[28] M. Limbäck, T. Andersson, in: Zirconium in the $\mathrm{Nu}-$ clear Industry: Eleventh International Symposium, ASTM STP 1295, pp. 448-468.

[29] N. E. Hoppe, in: Proceedings of the ANS-ENS International Topical Meeting on LWR Fuel Performance, Avignon, France, pp. 157-172.

[30] Y. Rashid, R. Dunham, R. Montgomery, Fuel Analysis and Licensing Code: FALCON MOD01, Technical Report EPRI 1011308, Electric Power Research Institute, 2004.

[31] P. M. Gilmore, H. H. Klepfer, J. M. Sorensen, EPRI PWR Fuel Cladding Corrosion (PFCC) Model Volume 1: Theory and Users Manual, Technical Report TR105387-V1, EPRI, December 1995.

[32] G. Schanz, Recommendations and supporting information on the choice of Zirconium oxidation models in severe accident codes, Technical Report FZKA 6827, SAM-COLOSS-P043, Forschungszentrum Karlsruhe GmbH, Karlsruhe, Germany, 2003.

[33] D. Stafford, Journal of Nuclear Materials 466 (2015) $362-372$.

[34] M. R. Tonks, P. C. Millett, P. Nerikar, S. Du, 
Table 3: Microstructure state variables for the cladding

\begin{tabular}{|l|c|c|c|}
\hline State Variable & Readiness & Modeling approach & Experimental approach \\
\hline 1. Average grain size & Medium & MD, PFM & OM, SEM, EBSD \\
2. Line dislocation density & Low & MD, DD, PFM, RT & TEM, XRD, APT, FIM \\
3. Void density & Low & MD, DD, PFM & SEM, TEM \\
4. Dislocation loop density & Low & MD, DD, PFM, RT & TEM, XRD, APT, FIM \\
5. H conc. in solution & Medium & DFT, MD, KMC, PFM & ECP, UT, SIMS, EELS \\
6. Hydride frac. & Medium & DFT, MD, PFM & OM, SEM, TEM, EPMA, EELS \\
7. 2nd phase particle frac. & Low & DFT, MD, KMC, PFM & OM, SEM, TEM, EPMA, EELS \\
\hline
\end{tabular}

Table 4: Property/microstructure relationships for the cladding. Note that the state variable numbers refer to Table 3

\begin{tabular}{|l|c|c|c|c|}
\hline Property & State vars. & Readiness & Modeling approach & Experimental approach \\
\hline Elastic constants & $1-7$ & Low & DFT, MD, SM & TT, UT, RUS, NI \\
Yield stress & $1-4,6,7$ & Low & MD, DD, SM & TT, NI \\
Thermal conductivity & $1-7$ & Low & DFT, HC & LF, DSC, VM, DIL \\
Fracture toughness & $1-4,5-7$ & Low & DFT, MD, PFM, SM & CIT, CTT, NI \\
Dimensional change & $1-4,6,7$ & Low & PFM, SM, RT & VM, DIL, XRD \\
\hline
\end{tabular}

D. Andersson, C. R. Stanek, D. Gaston, D. Andrs, R. Williamson, Journal of Nuclear Materials 440 (2013) 193-200.

[35] M. R. Tonks, X.-Y. Liu, D. Andersson, D. Perez, A. Chernatynskiy, G. Pastore, C. R. Stanek, R. Williamson, Journal of Nuclear Materials 469 (2016) 89-98.

[36] R. Jackson, A. Murray, J. Harding, C. Catlow, Philosophical Magazine A 53 (1986) 27-50.

[37] I. Hobson, R. Taylor, J. Ainscough, Journal of Physics D: Applied Physics 7 (1974) 1003.

[38] A. T. Nelson, D. R. Rittman, J. T. White, J. T. Dunwoody, M. Kato, K. J. McClellan, Journal of the American Ceramic Society 97 (2014) 3652-3659.

[39] B. Willis, Acta Crystallographica Section A: Crystal Physics, Diffraction, Theoretical and General Crystallography 34 (1978) 88-90.

[40] D. Bevan, I. Grey, B. Willis, Journal of Solid State Chemistry 61 (1986) 1-7.

[41] D. Andersson, G. Baldinozzi, L. Desgranges, D. Conradson, S. Conradson, Inorganic chemistry 52 (2013) $2769-2778$

[42] J. Ramirez, M. Stan, P. Cristea, Journal of nuclear materials 359 (2006) 174-184.

[43] C. Newman, G. Hansen, D. Gaston, Journal of Nuclear Materials 392 (2009) 6-15.

[44] J. Higgs, B. Lewis, W. Thompson, Z. He, Journal of Nuclear Materials 366 (2007) 99-128.

[45] H. Matzke, Journal of the Chemical Society, Faraday Transactions 2: Molecular and Chemical Physics 83 (1987) 1121-1142.

[46] H. Matzke, Radiation Effects 53 (1980) 219-242.

[47] C. Catlow, Radiation Effects 53 (1980) 127-132.

[48] R. G. J. Ball, R. W. Grimes, J. Chem. Soc., Faraday Trans. 86 (1990) 1257-1261.

[49] P. V. Nerikar, X.-Y. Liu, B. P. Uberuaga, C. R. Stanek, S. R. Phillpot, S. B. Sinnott, J. Phys. Condens. Matter 21 (2009) 435602.

[50] R. Grimes, C. Catlow, Philosophical Transactions of the Royal Society of London A: Mathematical, Physi- cal and Engineering Sciences 335 (1991) 609-634.

[51] J.-P. Crocombette, J. Nucl. Mater. 305 (2002) 29-36.

[52] M. Freyss, N. Vergnet, T. Petit, J. Nucl. Mater. 352 (2006) 144-150.

[53] D. A. Andersson, B. P. Uberuaga, P. V. Nerikar, C. Unal, C. R. Stanek, Phys. Rev. B 84 (2011) 054105.

[54] A. E. Thompson, C. Wolverton, Phys. Rev. B 84 (2011) 134111.

[55] Y. Yun, P. M. Oppeneer, H. Kim, K. Park, Acta Mater. 57 (2009) 1655-1659.

[56] W. Miekeley, F. Felix, Journal of Nuclear Materials 42 (1972) 297-306.

[57] P. V. Nerikar, K. Rudman, T. G. Desai, D. Byler, C. Unal, K. J. McClellan, S. R. Phillpot, S. B. Sinnott, P. Peralta, B. P. Uberuaga, et al., Journal of the American Ceramic Society 94 (2011) 1893-1900.

[58] K. Ahmed, C. A. Yablinsky, A. Schulte, T. Allen, A. El-Azab, Modelling and Simulation in Materials Science and Engineering 21 (2013) 065005.

[59] K. Ahmed, J. Pakarinen, T. Allen, A. El-Azab, Journal of Nuclear Materials 446 (2014) $90-99$.

60] M. R. Tonks, Y. Zhang, X. Bai, P. C. Millett, Materials Research Letters 2 (2014) 23-28.

61] K. Ahmed, T. Allen, A. El-Azab, Journal of Materials Science (2015) 1-17.

[62] M. R. Tonks, Y. Zhang, A. Butterfield, X.-M. Bai, Modelling and Simulation in Materials Science and Engineering 23 (2015) 045009.

[63] X.-M. Bai, Y. Zhang, M. R. Tonks, Acta Materialia 85 (2015) 95-106.

[64] K. Forsberg, A. R. Massih, J. Nucl. Mater. 135 (1985) 140-148.

[65] K. Forsberg, A. R. Massih, J. Nucl. Mater. 127 (1985) $141-145$.

[66] J. A. Turnbull, C. A. Friskney, J. R. Findlay, F. A. Johnson, A. J. Walter, J. Nucl. Mater. 107 (1982) 168184.

[67] R. J. White, M. O. Tucker, J. Nucl. Mater. 118 (1983) $1-38$.

[68] P. Nerikar, T. Watanabe, J. S. Tulenko, S. R. Phillpot, 
S. B. Sinnott, J. Nucl. Mater. 384 (2009) 61-69.

[69] B. Dorado, B. Amadon, M. Freyss, M. Bertolus, Phys. Rev. B 79 (2009) 235125.

[70] B. Dorado, G. Jomard, M. Freyss, M. Bertolus, Phys. Rev. B 82 (2010) 035114

[71] B. Dorado, P. Garcia, Phys. Rev. B 87 (2013) 195139.

[72] D. A. Andersson, M. R. Tonks, L. Casillas, S. Vyas, P. Nerikar, B. P. Uberuaga, C. R. Stanek, Journal of Nuclear Materials 462 (2015) $15-25$.

[73] D. A. Andersson, P. Garcia, X.-Y. Liu, G. Pastore, M. Tonks, P. Millett, B. Dorado, D. R. Gaston, D. Andrs, R. L. Williamson, R. C. Martineau, B. P. Uberuaga, C. R. Stanek, J. Nucl. Mater. 451 (2014) 225-242.

[74] B. Dorado, D. A. Andersson, C. R. Stanek, M. Bertolus, B. P. Uberuaga, G. Martin, M. Freyss, P. Garcia, Phys. Rev. B 86 (2012) 035110.

[75] J.-P. Crocombette, Phys. Rev. B 85 (2012) 144101.

[76] Y. Yun, H. Kim, H. Kim, K. Park, J. Nucl. Mater. 378 (2008) 40-44.

[77] Y. Yun, O. Eriksson, P. M. Oppeneer, H. Kim, K. Park, J. Nucl. Mater. 385 (2009) 364-367.

[78] E. Vathonne, J. Wiktor, M. Freyss, G. Jomard, M. Bertolus, Journal of Physics: Condensed Matter 26 (2014) 325501

[79] R. A. Jackson, A. D. Murray, J. H. Harding, C. R. A. Catlow, Phil. Mag. A 53 (1986) 27-50.

[80] C. R. A. Catlow, Proc. R. Soc. Lond. A 364 (1978) 473-497.

[81] R. W. Grimes, C. R. A. Catlow, Phil. Trans. R. Soc. Lond. A 335 (1991) 609-634.

[82] R. G. J. Ball, R. W. Grimes, J. Chem. Soc., Faraday Trans. 86 (1990) 1257-1261.

[83] R. G. J. Ball, R. W. Grimes, J. Nucl. Mater. 188 (1992) $216-221$.

[84] K. Govers, S. Lemehov, M. Verwerft, J. Nucl. Mater. 405 (2010) 252-260

[85] S. T. Murphy, A. Chartier, L. Van Brutzel, J.-P. Crocombette, Phys. Rev. B 85 (2012) 144102

[86] S. Nicoll, H. Matzke, C. R. A. Catlow, J. Nucl. Mater. 226 (1995) 51-57.

[87] A. E. Thompson, C. Wolverton, Phys. Rev. B 87 (2013) 104105

[88] X.-Y. Liu, B. P. Uberuaga, D. A. Andersson, C. R. Stanek, K. E. Sickafus, Appl. Phys. Lett. 98 (2011) 151902.

[89] T. Petit, M. Freyss, P. Garcia, P. Martin, M. Ripert, J.-P. Crocombette, F. Jollet, J. Nucl. Mater. 320 (2003) 133-137.

[90] T. Petit, G. Jomard, C. Lemaignan, B. Bigot, A. Pasturel, J. Nucl. Mater. 275 (1999) 119-123.

[91] X.-Y. Liu, B. Uberuaga, P. Nerikar, C. Stanek, K. Sickafus, Nucl. Instrum. Methods Phys. Res., Sect. B 268 (2010) 3014-3017.

[92] H. Y. Geng, Y. Chen, Y. Kaneta, M. Kinoshita, Q. Wu, Phys. Rev. B 82 (2010) 094106

[93] P. C. Millett, Y. Zhang, M. R. Tonks, S. Biner, Journal of Nuclear Materials 440 (2013) 435 - 439

[94] P. V. Nerikar, D. C. Parfitt, L. A. Casillas Trujillo, D. A. Andersson, C. Unal, S. B. Sinnott, R. W. Grimes, B. P. Uberuaga, C. R. Stanek, Phys. Rev. B 84 (2011) 174105.

[95] P. C. Millett, M. R. Tonks, S. Biner, Journal of Nuclear Materials 424 (2012) $176-182$

[96] P. C. Millett, M. R. Tonks, S. Biner, L. Zhang, K. Chockalingam, Y. Zhang, Journal of Nuclear Mate- rials 425 (2012) 130 - 135. Microstructure Properties of Irradiated Materials.

[97] P. C. Millett, Y. Zhang, D. Andersson, M. R. Tonks, S. Biner, Journal of Nuclear Materials 430 (2012) 44 49.

[98] X.-Y. Liu, D. Andersson, Journal of Nuclear Materials $462(2015) 8-14$.

[99] E. Moore, L. R. Corrales, T. Desai, R. Devanathan, Journal of Nuclear Materials 419 (2011) 140-144.

[100] D. Schwen, R. Averback, Journal of Nuclear Materials 402 (2010) $116-123$.

[101] S. Hu, C. H. Henager Jr., H. L. Heinisch, M. Stan, M. I. Baskes, S. M. Valone, J. Nucl. Mater. 392 (2009) 292-300.

[102] P. Garcia, G. Martin, C. Sabathier, G. Carlot, A. Michel, P. Martin, B. Dorado, M. Freyss, M. Bertolus, R. Skorek, J. Noirot, L. Noirot, O. Kaitasov, S. Maillard, Nucl. Intrum. Meth. B 277 (2012) 98-108.

[103] Y. Li, S. Hu, R. Montgomery, F. Gao, X. Sun, Nuclear Instruments and Methods in Physics Research Section B: Beam Interactions with Materials and Atoms 303 (2013) 62 - 67. Proceedings of the 11th Computer Simulation of Radiation Effects in Solids (COSIRES) Conference Santa Fe, New Mexico, USA, July 24-29, 2012.

[104] D. Schwen, M. Huang, P. Bellon, R. Averback, Journal of Nuclear Materials 392 (2009) $35-39$.

[105] M. Huang, D. Schwen, R. Averback, Journal of Nuclear Materials 399 (2010) $175-180$

[106] K. Govers, C. Bishop, D. Parfitt, S. Lemehov, M. Verwerft, R. Grimes, Journal of Nuclear Materials 420 (2012) $282-290$

[107] M. Veshchunov, A. Boldyrev, A. Kuznetsov, V. Ozrin, M. Seryi, V. Shestak, V. Tarasov, G. Norman, A. Kuksin, V. Pisarev, D. Smirnova, S. Starikov, V. Stegailov, A. Yanilkin, Nuclear Engineering and Design 295 (2015) 116 - 126.

[108] L. Noirot, Nucl. Eng. Des. 241 (2011) 2099-2118.

[109] M. S. Veshchunov, V. E. Shestak, J. Nucl. Mater. 376 (2008) 174-180.

[110] M. Veshchunov, V. Ozrin, V. Shestak, V. Tarasov, R. Dubourg, G. Nicaise, Nucl. Eng. Des. 236 (2006) 179-200.

[111] M. S. Veshchunov, R. Dubourg, V. Ozrin, V. Shestak, V. Tarasov, J. Nucl. Mater. 362 (2007) 327-335.

[112] M. S. Veshchunov, J. Nucl. Mater. 277 (2000) 67-81.

[113] Y. Zhang, X.-Y. Liu, P. C. Millett, M. Tonks, D. A. Andersson, B. Biner, Journal of Nuclear Materials 430 (2012) 96-105.

[114] Y. Zhang, P. C. Millett, M. R. Tonks, X.-M. Bai, S. B. Biner, in: Proceedings of GLOBAL 2013: International Nuclear Fuel Cycle Conference-Nuclear Energy at a Crossroads.

[115] Y. Zhang, P. C. Millett, M. R. Tonks, X.-M. Bai, S. B. Biner, Journal of Nuclear Materials 452 (2014) 296303.

[116] P. Chakraborty, Y. Zhang, M. R. Tonks, Computational Materials Science 113 (2016) 38-52.

[117] P. Chakraborty, M. R. Tonks, G. Pastore, Journal of Nuclear Materials 452 (2014) 95-101.

[118] R. L. Williamson, D. A. Knoll, in: 20th International Conference on Structural Mechanics in Reactor Technology (SMiRT 20), Espoo (Helsinki), Finland.

[119] B. W. Spencer, H. Huang, J. Dolbow, J. D. Hales, in: 2014 LWR Fuel Performance Meeting - TopFuel, Sendai, Japan. 
[120] H. Huang, B. Spencer, J. Hales, Nuclear Engineering and Design 278 (2014) 515-528.

[121] J. W. Pang, W. J. Buyers, A. Chernatynskiy, M. D. Lumsden, B. C. Larson, S. R. Phillpot, Physical review letters 110 (2013) 157401.

[122] K. Govers, S. Lemehov, M. Hou, M. Verwerft, Journal of nuclear materials 366 (2007) 161-177.

[123] A. Chernatynskiy, C. Flint, S. Sinnott, P. SR, J Mat Sci 47 (2012) 7693-7702.

[124] T. Watanabe, S. B. Sinnott, J. S. Tulenko, R. W. Grimes, P. K. Schelling, S. R. Phillpot, Journal of Nuclear Materials 375 (2008) 388-396.

[125] J. P. Moore, D. L. Mcelroy, Journal of the American Ceramic Society 54 (1971) 40-46.

[126] K. Gofryk, S. Du, C. R. Stanek, J. C. Lashley, X.-Y. Liu, R. K. Schulze, J. L. Smith, D. J. Safarik, D. D. Byler, K. J. McClellan, B. P. Uberuaga, B. L. Scott, D. A. Andersson, Nat Commun 5 (2014) 4551.

[127] T. Watanabe, S. G. Srivilliputhur, P. K. Schelling, J. S. Tulenko, S. B. Sinnott, S. R. Phillpot, Journal of the American Ceramic Society 92 (2009) 850-856.

[128] S. Nichenko, D. Staicu, Journal of Nuclear Materials 433 (2013) 297-304.

[129] B. Deng, A. Chernatynskiy, S. Sinnott, S. Phillpot, J Nucl Mat 434 (2013) 203-209.

[130] C.-W. Lee, A. Chernatynskiy, P. Shukla, R. Stoller, S. Sinnott, S. Phillpot, Journal of Nuclear Materials 456 (2015) 253-259.

[131] P. C. Millett, D. Wolf, T. Desai, S. Rokkam, A. ElAzab, Journal of Applied Physics 104 (2008) 033512.

[132] S. Hu, C. H. Henager Jr., H. L. Heinisch, M. Stan, M. I. Baskes, S. M. Valone, Journal of Nuclear Materials 392 (2009) 292-300.

[133] P. C. Millett, M. Tonks, Journal of Nuclear Materials 412 (2011) 281-286.

[134] K. Chockalingam, P. C. Millett, M. R. Tonks, Journal of Nuclear Materials 430 (2012) 166-170.

[135] M. C. Teague, B. S. Fromm, M. R. Tonks, D. P. Field, JOM 66 (2014) 2569-2577.

[136] M. Teague, M. Tonks, S. Novascone, S. Hayes, Journal of Nuclear Materials 444 (2014) 161-169.

[137] A. Motta, A. Couet, C. RJ, Ann Rev Mat Res 45 (2015) 311-343.

[138] T.-W. Chiang, A. Chernatynskiy, M. J. Noordhoek, S. B. Sinnott, S. R. Phillpot, Computational Materials Science 98 (2015) 112-116.

[139] H. G. Kim, T. H. Kim, Y. H. Jeong, Journal of nuclear materials 306 (2002) 44-53.

[140] B. Puchala, A. Van der Ven, Phys. Rev. B 88 (2013) 094108

[141] A. Couet, A. T. Motta, A. Ambard, Corrosion Science 100 (2015) 73-84.

[142] X. Bai, Y. Zhang, M. Tonks, Phys. Chem. Chem. Phys. 15 (2013) 19438-19449.

[143] J. J. Kearns, J. Nucl. Mater. 22 (1967) 292-303.

[144] A. T. Motta, L. Q. Chen, JOM 64 (2012) 1403.

[145] J. Bair, M. A. Zaeem, M. Tonks, J. Nucl. Mater. 466 (2015) 12-20.

[146] R. S. Daum, D. W. Bates, D. A. Koss, A. T. Motta, in: Proceedings of the International Conference on Hydrogen Effects on Material Behaviour and Corrosion Deformation Interactions, Warrendale, PA: Minerals, Metals and Materials Society, 2003, Moran, WY, 2002, p. 249.

[147] T. Kubo, Y. Kobayashi, H. Uchikoshi, J. Nucl. Mater.
435 (2013) 222

[148] T. R. Allen, R. J. M. Konings, A. T. Motta, Comprehensive Nucl. Mater. 5 (2012) 49-68.

[149] C. Domain, R. Besson, A. Legris, Acta Mater. 50 (2002) 3513

[150] Y. Udagawa, M. Yamaguchi, H. Abe, N. Sekimura, T. Fuketa, Acta Mater. 58 (2010) 3927.

[151] P. A. Burr, S. T. Murphy, S. C. Lumley, M. R. Wenman, R. W. Grimes, Corros. Sci. 69 (2013) 1-4.

[152] S. C. Lumley, R. W. Grimes, S. T. Murphy, P. A. Burr, A. Chroneos, P. R. Chard-Tuckey, M. R. Wenman, Acta Mater. 79 (2014) 351.

[153] M. Christensen, W. Wolf, C. M. Freeman, E. Wimmer, R. B. Adamson, L. Hallstadius, P. E. Cantonwine, E. Mader, J. Phys.: Condens. Matter 27 (2015) 025402 .

[154] J. J. Kearns, J. Nucl. Mater. 43 (1972) 330-338.

[155] W. Zhu, W. R, G. Shu, P. Wu, H. Xiao, J. Phys. Chem. C 114 (2010) 22361.

[156] Y. Zhong, D. D. MacDonald, J. Nucl. Mater. 423 (2012) 87-92.

[157] A. Alajem, Solid State Phenomena 49-50 (1996) 281330.

[158] M. P. Puls, The Effect of Hydrogen and Hydrides on the Integrity of Zirconium Alloy Component, Springer, 2012 .

[159] Z. Zhao, M. Blat-Yrieix, J. P. Morniroli, A. legris, L. Thuinet, Y. Kihn, A. Ambard, L. Legras, J. of ASTM International 5 (2008) 1-20.

[160] L. Holliger, A. Legris, R. Besson, Phys. Rev. B 80 (2009) 094111.

[161] L. Thuinet, R. Besson, Intermetallics 20 (2012) 24

[162] G. J. C. Carpenter, Acta Metallurgica 26 (1978) 1225.

[163] Y. Zhang, X.-M. Bai, J. Yu, M. R. Tonks, M. J. Noordhoek, S. R. Phillpot, Acta Materialia 111 (2016) 357-365.

[164] J. C. Thomas, A. Van der Ven, Physical Review B 90 (2014) 224105.

[165] X. Ma, S. Q. Shi, C. Woo, L. Chen, Comp. Mater. Sci. 23 (2002) 283-290.

[166] Z. Xiao, M. Hao, X. Guo, G. Tang, S.-Q. Shi, J. Nucl. Mater. 459 (2015) 330-338.

[167] K. L. Murty, I. Charit, Progress in Nuclear Energy 48 (2006) 325-359.

[168] Z. Lu, M. J. Noordhoek, A. Chernatynskiy, S. B. Sinnott, S. R. Phillpot, Journal of Nuclear Materials 462 (2015) 147-159.

[169] Z. Lu, A. Chernatynskiy, M. J. Noordhoek, S. B. Sinnott, S. R. Phillpot, Journal of Nuclear Materials 467 (2015) 742-757.

[170] G. Carpenter, R. Murgatroyd, A. Rogerson, J. Watters, Journal of Nuclear Materials 101 (1981) 28-37.

[171] A. Rogerson, R. Zee, Journal of Nuclear Materials 151 (1987) 81-83.

[172] F. Christien, A. Barbu, Journal of Nuclear Materials 393 (2009) 153-161.

[173] R. A. Lebensohn, C. S. Hartley, C. N. Tomé, O. Castelnau, Philosophical Magazine 90 (2010) 567-583.

[174] G. Subramanian, C. Tome, A fully crystallographic coupled formulation of irradiation growth and climbcontrolled-glide. Incorporation into the polycrystal code VPSC, Technical Report LA-UR-13-28204, Los Alamos National Laboratory, 2013.

[175] S. Golubov, A. Barashev, R. Stoller, On the origin of radiation growth in HCP crystals, Technical Report 
ORNL/TM-2011/473, Oakridge National Laboratory, 2011.

[176] G. Subramanian, D. Perez, B. P. Uberuaga, C. N. Tomé, A. F. Voter, Physical Review B 87 (2013) 144107.

[177] A. Alfonsi, C. Rabiti, D. Mandelli, J. Cogliati, R. Kinoshita, in: Proceeding of M\&C2013 International Topical Meeting on Mathematics and Computation.

[178] R. Williamson, K. Gamble, D. Perez, S. Novascone, G. Pastore, R. Gardner, J. Hales, W. Liu, A. Mai, Nuclear Engineering and Design 301 (2016) 232-244. 